Published in final edited form as:

Epileptic Disord. 2015 June ; 17(2): 101-116. doi:10.1684/epd.2015.0739.

\title{
Cognitive impairment in Epilepsy: The Role of Network Abnormalities
}

\author{
Gregory L. Holmes, MD \\ Department of Neurological Sciences, University of Vermont College of Medicine, Burlington, \\ Vermont
}

\section{Abstract}

The challenges to individuals with epilepsy extend far beyond the seizures. Co-morbidities in epilepsy are very common and are often more problematic to individuals than the seizures themselves. In this review, the pathophysiological mechanisms of cognitive impairment are discussed. While etiology of the epilepsy has a significant influence on cognition there is increasing evidence that prolonged or recurrent seizures can cause or exacerbate cognitive impairment. Alterations in signaling pathways and neuronal network function play a major role in both the pathophysiology of epilepsy and the epilepsy comorbidities. However, the biological underpinnings of cognitive impairment can be distinct from the pathophysiological processes that cause seizures.

\section{Keywords}

memory; cognition; place cells; replay; reactivation; oscillations; rate coding; temporal coding; accelerated forgetting

\section{Introduction}

\begin{abstract}
Although seizures are the most striking clinical manifestation of the epilepsies, individuals with epilepsy are at risk not only for seizures, but also for a myriad of health problems that occur in people with epilepsy at a higher rate than would be expected by chance (Committee on the Public Health Dimensions of the Epilepsies et al., 2012). Common co-morbidities that occur in epilepsy include cognitive dysfunction, such as memory, attention, or processing difficulties; mental health conditions, including depression or anxiety; and somatic co-morbidities, such as sleep disorders and migraines. Epilepsy comorbidities are common and often severe. For many individuals with epilepsy the co-morbidities are more burdensome than the seizures.
\end{abstract}

Among the co-morbidities associated with epilepsy, cognitive abnormalities are among the most common and troublesome. In people with epilepsy there is an associated high rate of cognitive difficulties that compromise educational progress and achievement throughout life

Corresponding Author: Gregory L. Holmes, M.D., University of Vermont College of Medicine, 95 Carrigan Drive, Neurological Sciences - Stafford 118, Burlington, VT 05405, UNITED STATES, Phone: 802.656.4588, Fax: 802.656.5678, gregory.holmes@uvm.edu. 
(Berg et al., 2008). In addition to a higher incidence of low IQs (Farwell et al., 1985), in about half of children with epilepsy there is an identified discrepancy between IQ and achievement (Fastenau et al., 2008). Children who have poorly controlled (pharmacoresistant) seizures are more likely to have lower IQ scores than children with well controlled seizures (Berg et al., 2012). Adults with chronic epilepsy are also vulnerable for cognitive regression (Thompson and Duncan, 2005; Bell et al., 2011).

Both children and adults with epilepsy frequently complain of memory disorders (Thompson and Corcoran, 1992; Butler and Zeman, 2008). Individuals with epilepsy may have transient epileptic amnesia, in which the sole or main feature of the epilepsy is episodic amnesia, accelerated long-term forgetting, in which newly acquired memories fade over days to weeks and remote memory impairment in which autobiographical or public facts are forgotten (Butler and Zeman, 2008). Accelerated long-term forgetting is a condition where individuals learn and initially retain information normally, but forget the information at an unusually rapid rate (Blake et al., 2000). Accelerated forgetting has been demonstrated in both adults (Butler et al., 2009) and children (Martinos et al., 2012). Both accelerated forgetting and remote memory impairment is seen primarily in individuals with temporal lobe epilepsy (Butler et al., 2009; Muhlert et al., 2011).

Much of the cognitive impairment that occurs in people with epilepsy is related to the underlying etiology of the epilepsy. Both acquired disorders such as trauma, hypoxia, ischemia, and mesial temporal sclerosis secondary to prolonged seizures and genetic disorders, including tuberous sclerosis, fragile X, Rett, and Dravet syndromes can lead to significant cognitive impairment in addition to causing epilepsy. In addition to these static deficits caused by the underlying etiology, cognitive impairments can be more dynamic or transient, occurring as a result of the seizures, EEG interictal spikes (IIS) and antiepileptic drugs. In many patients there may be a combination of reasons for the cognitive impairment. Both the dynamic and static effects contributing to cognitive impairment will be reviewed. While some antiepileptic drugs have pronounced effects on cognition (Hermann et al., 2010), this topic will not be discussed here.

While there have recently been increased efforts to recognize epilepsy co-morbidities, our understanding of the pathophysiological mechanisms responsible for the co-morbidities lags far behind our knowledge of the mechanism of epilepsy. In this review, recent advances in the neurobiology of cognitive impairment are reviewed with emphasis on network changes.

\section{Dynamic Effects on Cognition: Seizures and IIS}

Recurrent seizures, an essential component of epilepsy, can seriously affect cognition acutely. In addition to the obvious incapacities occurring during the seizure, the post-ictal state usually is a period of decreased cognitive ability. After the behavioral symptoms of lethargy and inattention subside, lingering cognitive deficits may persist for minutes to days depending on the type and severity of the seizure. Following generalized seizures, rats have spatial memory deficits in the Morris water maze with the duration of the impairment exceeding the length of the seizure (Boukhezra et al., 2003). When seizures occur frequently there is a cumulative degradation in spatial performance (Lin et al., 2009). While the exact reason for the cause of the post-ictal impairment has not been established, it is known that 
recurrent seizures result in impairment in long-term potentiation (LTP), the precision and timing of place cells that underlie spatial memory (Zhou et al., 2007a; Zhou et al., 2007c), and the power and frequency of theta oscillations that represent the orderly communication of networks, all factors which could negatively affect cognition.

In addition to seizures, IIS can result in cognitive impairment in both rats and humans (Fig. 1). Following intrahippocmapal pilocarpine infusion, rats develop IIS that result in transient impairment in the delayed-match-to-sample test, a hippocampal-dependent operant behavior task (Kleen et al., 2010). Hippocampal IIS that occur during memory retrieval strongly impairs performance. However, IIS that occur during memory encoding or memory maintenance do not affect performance. In a similar study of people with temporal lobe epilepsy (TLE) who have bilateral depth electrodes implanted into their hippocampi for preoperative seizure localization, IIS cause transient memory impairment (Krauss et al., 1997; Kleen et al., 2013). Hippocampal IIS occurring during the memory retrieval period decreases the likelihood of a correct response on a short-term memory test, the Sternberg task, when they occur bilaterally in both temporal lobes or in the temporal lobe contralateral to the seizure focus (Kleen et al., 2013). Because patients with TLE often have impaired memory in the epileptic hippocampus and require the non-epileptic hippocampus for memory, IIS arising from the damaged epileptic hippocampus has little effect on memory while IIS involving the intact non-epileptic hippocampus interfere with memory retrieval. As with rodents with TLE, patients with TLE and IIS have specific deficits in retrieval of memory while sparing other forms of memory.

IIS in rodents, similar to seizures, result in a sustained reduction of action potentials (APs) in the hippocampus for up to two seconds following IIS (Zhou et al., 2007b). Furthermore, when occurring in flurries, defined as four or more IIS occurring within $10 \mathrm{sec}$, IIS can reduce APs firing for up to six seconds. The widespread inhibitory wave immediately following IIS can also reduce the power of gamma $(>30 \mathrm{~Hz})$ oscillations and other oscillatory signals in the hippocampus (Urrestarazu et al., 2006). As will be discussed further, since gamma oscillations are closely coupled with ongoing learning and memory function, disruption in oscillations may contribute to cognitive deficits.

In adults with IIS, the cognitive effects are typically transient and may have little long-term consequences in cognitive function, unless they occur very frequently. However, in the immature brain, the effects of IIS also have long-term adverse effects on developing neural circuits. In interesting studies from the 1980's, IIS were elicited by either penicillin (Baumbach and Chow, 1981) or bicuculline (Ostrach et al., 1984) through focal application on the striate cortex of the occipital lobe of immature rabbits. IIS occurred for 6-12 hours following each drug application which were given daily starting from postnatal $(\mathrm{P})$ day 8-9 and continued for 3-4 weeks. None of the rabbits had behavioral or electrographic seizures. In single-unit recordings from the lateral geniculate nucleus, superior colliculus and occipital cortex ipsilateral to the occipital lobe with the IIS, an abnormal distribution of receptive field types was seen, whereas normal recording were present in the contralateral hemisphere. Remarkably, this finding is age-dependent. Adult rabbits with similarly induced IIS have a normal distribution of receptive field types, highlighting the vulnerability of critical developmental periods to cumulative IIS effects over time. Focally-induced IIS by 
stereotaxic injections of bicuculline in the prefrontal cortex (PFC) in rat pups for several days also result in long-standing increases in short term plasticity in the PFC and marked inattentiveness during a spatial memory task, when the rats are tested as adults at a time when IIS are no longer present (Hernan et al., 2014).

\section{Static Effects of Seizures on Cognition}

Individuals with prolonged seizures lasting 30 minutes or more, a condition termed status epilepticus (SE), or frequent recurrent seizures are at particular risk for brain injuries that can result in cognitive impairment. While the cause of the seizure clearly contributes to cognitive outcome, there is abundant evidence both in humans and animal models that seizures independent of etiology worsen cognitive outcome (Kleen et al., 2012). SE also may set in motion the process of epileptogeneis resulting in spontaneous seizures (Choy et al., 2014). During SE, there are marked increases in excitation and decreases in inhibition resulting in a cascade of effects that occur during and after the seizure which induce both morphological and functional changes in the brain (Kleen et al., 2012)(Fig. 2). The cognitive deficits occurring as a result of the SE can be exacerbated by the spontaneous seizures that occur following SE. Many of these SE-induced changes, such as oxidative stress, apoptosis, neurogenesis, changes in growth factors such as BDNF and inflammation occur repeatedly with spontaneous seizures.

When studied weeks or months following SE, rats are impaired in spatial memory tasks such as the Morris water maze (Liu et al., 2003; Lenck-Santini and Holmes, 2008), radial arm maze (Sayin et al., 2004) and novel object exploration test (Chauviere et al., 2009). Paralleling this development of cognitive impairment, a number of morphological and physiological changes occur in brain networks as a result of SE. In the adult rat, neuronal loss occurs throughout the hippocampus, parahippocampus and entorhinal cortex with loss of both principal cells and interneurons. In addition to cell death, SE in the adult brain leads to synaptic reorganization, with aberrant growth (sprouting) of granule cell axons (the socalled mossy fibers) into CA3. Sprouting and new synapse formation occurs in other brain regions as well, such as the CA1 pyramidal neurons, where the newly formed synapses have an increase in glutamatergic spontaneous synaptic currents. Seizures can result in neurogenesis in the dentate gyrus and such ectopic granule cells may be abnormally positioned in the hilus and contribute to enhanced excitability in hippocampal networks (Koyama et al., 2012; Myers et al., 2013). The degree of seizure-induced damage and cognitive impairment is related to the severity of the seizures. There is a continuum with SE causing more profound morphological and physiological changes in the brain than briefer seizures. Teasing out which seizure-induced change is responsible for the cognitive difficulties is difficult because seizures, regardless of whether it is SE or a briefer seizure, result in a multitude of changes that occur simultaneously. It appears highly unlikely that one single factor accounts for cognitive impairment in epilepsy. While it would be logical to believe that cognitive impairment is directly related to cell loss, there is poor correlation between cell loss and cognition in rodents (Faure et al., 2014).

Recurrent seizures as a result of a variety of etiologies can lead to persistent decreases in GABA currents in the hippocampus (Isaeva et al., 2006) and neocortex (Isaeva et al., 2010), 
enhanced excitation in the neocortex (Isaeva et al., 2010), impairment in spike frequency adaptation (Villeneuve et al., 2000), marked reductions in afterhyperpolarizing potentials following spike trains (Villeneuve et al., 2000), impaired LTP (Zhou et al., 2007c; Karnam et al., 2009a; Isaeva et al., 2013), alterations in theta power (Karnam et al., 2009b) and precision in place cell firing precision and stability (Karnam et al., 2009b). Similar to SE, rats with recurrent spontaneous seizures have behavioral deficits of spatial cognition in the water maze (Karnam et al., 2009b; Lugo et al., 2014), non-match to sample task (Kleen et al., 2011b) and impaired auditory discrimination (Neill et al., 1996) and reduced behavioral flexibility (Kleen et al., 2011a).

The morphological changes and functional changes in the brain following SE or recurrent seizures result in changes in neural circuitry that directly affect the ability of the affected structures to process information normally. Such changes in neural circuitry can alter rate coding, where information is conveyed through the firing frequency of single neurons, as well as temporal coding, where information flow is achieved through the temporal interaction of multiple neurons firing. Errors in both rate and temporal coding are prominent in the chronic epilepsies. Such error in coding may be even more detrimental in developing animals where oscillations drive normal circuit formation and stabilization.

\section{Aberrant Rate Coding in Epilepsy}

The relationship between SE-induced network reorganization and cognitive dysfunction is best illustrated by single unit recordings in freely moving rats. A subset of neurons in the hippocampus, called place cells, elicit APs (APs) that correspond to the animal's location within its environment (O'Keefe and Dostrovsky, 1971). Specifically, these hippocampal pyramidal neurons selectively discharge when the animal enters certain locations of the environment, called the cells' firing field (Fig. 3). Field location, size and shape are specific to each cell and each environment and fields tend to cover the surface of the environment homogeneously when a large number of neurons are being recorded simultaneously. For a given environment, the fields remain unchanged, even between exposures separated by months. Since there is a relationship between place cell activity and the ongoing spatial behavior of rats, it is believed that such signals provide the animal with a spatial representation in order to navigate efficiently within the environment. These cells provide a very useful surrogate of spatial memory. Adult rats that have experienced SE and have impaired learning in the water maze have defective place cells (Liu et al., 2003; LenckSantini and Holmes, 2008). Place cells from the SE rats have less precise firing fields and less stable firing fields from session to session (Liu et al., 2003).

Likewise, recurrent seizures during the first weeks of life cause impairment in spatial cognition with poor performance in the water maze and radial arm maze and impaired hippocampal LTP (Karnam et al., 2009b). Similar to rats following SE, these rats have substantial deficits in APs firing with impaired place cell precision and reduced place cell stability. These results show that recurrent seizures during early development are associated with significant impairment in spatial learning and that these deficits are paralleled by deficits in the development of place cells. 


\section{Aberrant Temporal Coding}

In addition to abnormalities in rate coding, epilepsy can lead to disruptions in temporal coding. Network oscillations underlie the temporal binding of spatially distributed neuronal populations necessary for cognitive processing. Precise temporal organization of neuronal firing activity is believed to be a fundamental aspect of normal information processing. Recent studies support the idea that aberrant brain connectivity through alterations in oscillatory and impaired temporal coding has marked adverse effects on cognition in epilepsy.

The synchronous rhythms arising from fluctuations in postsynaptic inhibitory and excitatory potentials provide a mechanism for temporal coordination of neural activity in both local and long distance networks. As these neuronal ensembles oscillate, they effectively turn on and turn off their ability to send or receive information (Buzsaki and Draguhn, 2004; Womelsdorf et al., 2007). For information to be transferred from one neuronal group to another, the transmitting neurons must be excitable at the same time that the receiving group is excitable. Thus synchronization coordinates communication among neurons and thereby can support the dynamic configuration of functional networks (Fries, 2005; Womelsdorf et al., 2007).

Oscillations in the theta $(4-12 \mathrm{~Hz})$ and gamma $(30-90 \mathrm{~Hz})$ ranges have been extensively studied in the rat hippocampus and have led to insight into how oscillations can coordinate cellular processes related to memory. Theta rhythm is involved in mnemonic function of the hippocampus (McNaughton et al., 2006; Lega et al., 2012) whereas gamma oscillations correlate with perception processing or perceiving of sensory information (Gray and Viana Di, 1997), attention (Benchenane et al., 2011) and memory (Montgomery and Buzsaki, 2007).

Precise encoding of information in the hippocampus is dependent on theta oscillation; information arriving in the absence of normal theta activity is not encoded with the same degree of precision as when theta is present (Buzsaki, 2002). Additionally, the phase of theta is critical in learning and memory: tetanic stimulation in CA1 produces long-term potentiation (LTP) when administered at the peak of theta and long-term depression (LTD) when delivered at the trough (Hyman et al., 2003). Hippocampal theta frequency is also strongly modulated by running speed, with a positive correlation between running speed and theta frequency (Richard et al., 2013).

The importance of precision of the theta oscillation in spatial cognition was demonstrated by McNaughton et al. (McNaughton et al., 2006) who blocked theta transmission into the hippocampus by inactivating the septum with tetracaine in animals trained in the Morris water maze. Rats with no hippocampal theta showed no initial learning in the maze. When hippocampal theta was restored, using the supramammillary area theta frequency to trigger electrical stimulation of the fornix, initial learning was restored. Interestingly, when the electrically-induced theta occurred at a frequency different from the rat's normal theta frequency, learning was impaired compared to stimulation occurring at the frequency 
generated in the supramammillary area. These results demonstrate that the precise endogenous frequency of theta rhythm is necessary for optimal hippocampal function.

An important link between oscillations and cortical computations is the observation that oscillatory rhythms in the theta bandwidths provide synchronization of APs (O'Keefe and Recce, 1993). As a consequence, neurons participating in the same oscillatory rhythm synchronize their discharges with very high precision. An important example of this is the relationship between place cells and theta. In the hippocampus, place cells are not only characterized by their location-specific firing, but by their precise temporal firing relationship with hippocampal theta (Skaggs et al., 1996). When the rat enters in the cell's firing field APs will fire preferentially on the negative phase of the CA1 recorded theta cycle (Fig. 3). As the rat crosses the field, the cells fire earlier on successive theta waves, a phenomenon called phase precession (O'Keefe and Recce, 1993). Because of this characteristic, two cells with partially overlapping fields will fire at a specific, but slightly different, phase of the ongoing theta cycle. Their relative firing interval will be constant and directly related to the distance separating their fields (Fig. 3). As a result, the sequence and timing of events experienced by the animal is encoded: the time difference between APs is observed on a large time scale ( $\mathrm{T}$, the time it takes to get from field A to field $\mathrm{B}$ ) and also in the order of tens of milliseconds ( $t$ ). The firing sequences of cell assemblies observed in the running time are compressed in a time window short enough to induce long-term potentiation (LTP)-like synaptic changes. Using these measurements, a time compression index can be defined, for all possible pairs of cells, as the ratio of two spike timing measures: i) the time necessary for the animal to go from one field to the other; and ii) the equivalent compressed time in the theta domain, i.e., the time lag between the spikes of the two corresponding place cells within one theta cycle. Such coding of distance versus time provides the rat with a spatial representation of the environment in order to navigate efficiently within the environment. Abnormalities in temporal coding of place cell firing are associated with impaired memory (Robbe and Buzsaki, 2009)

Changes in theta oscillations and temporal coding of AP in the hippocampus occur in rats with epilepsy. Following SE, rats have reduced theta power which correlates with deficits in hippocampus-dependent spatial memory tasks (Chauviere et al., 2009). Following SE there are marked abnormalities in phase precession and impaired time compression of firing among pairs of neurons with parallel deficits in the Morris water maze task (Lenck-Santini and Holmes, 2008) (Fig. 3).

Along with impairment in phase precession in rats with epilepsy, speed/theta frequency correlation coefficients and regression slopes are abnormal compared to control animals (Richard et al., 2013) (Fig. 3). This impaired speed/theta frequency relationship is directly related to performance in task of spatial memory, indicating that in rats with epilepsy there is a deficit in the integration of locomotor information into memory processes. While the reason for the impaired memory in rats with impaired speed/theta frequency correlations is not known, a reasonable hypothesis is that since theta modulates place cell AP firing, if theta frequency does not increase with running speed fewer APs will fire as the animal transverses the firing field. The impaired speed:theta relationship coupled with impaired phase precession show that rats with epilepsy have substantially impaired temporal coding of 
information and that these impairments in coding are associated with deficits in spatial cognition.

Sequential reactivation of neurons occurs after spatial experience, for example after the rat has explored a new environment (Foster and Wilson, 2006). This reactivation, termed replay, has a unique form, in which recent episodes of spatial experience are replayed in a temporally reversed order during a physiological sharp-wave ripple (SWR) (Carr et al., 2012). Transient gamma synchronization serves as a clocking mechanism for SWR and enables coordinated memory reactivation across the hippocampal network. This replay of APs has been suggested to aid in consolidation of memory. In addition, there is data indicating that SWRs are also important in learning the task (Jadhav et al., 2012; Singer et al., 2013). Whereas following a task cells fire rapidly in reverse order from their occurrence during the task, when SWRs occur at decision points the cells fire in the order they occur during the task. Replay suggests online activation helps in planning, decision making, and consolidating memories of recent events. In addition to the online activation of cells during behavior, consolidation of memory also likely requires offline reactivation of behavioral patterns of neuronal activity during sleep. Sleep reactivation in the hippocampus is believed to aid in the consolidation of memories by the repetition of coordinated firing, which is communicated to neocortical brain regions to become enduring memories (Wilson and McNaughton, 1994). Studies have shown that the reactivation of neural patterns predict spatial memory performance (Dupret et al., 2010; Singer et al., 2013) while transient disruptions of neural activity during reactivation periods result in poor performance (EgoStengel and Wilson, 2010). Rats with epilepsy performing in a spatial task have less neuronal reactivation, demonstrating impaired online processing of spatial memory (Tyler et al., 2012). During sleep rats with TLE have place cells with more negatively correlated firing of APs than controls, suggesting an "undoing" of functional relationships between neurons activated by behavior (Titiz et al., 2014). These negative correlations may indicate an overactive system in TLE rats for accelerated forgetting, as seen in patient with TLE (Butler and Zeman, 2008).

In addition to modulating APs locally, oscillations have a major role in modulating neuronal firing at distant sites. Coherence is a measure of synchronization or coupling between two EEG signals and is based mainly on the conformity of phase differences between the EEG signals. High coherence values are taken as a measure of strong connectivity between the brain regions that produce the compared EEG signals. Firing of APs across distant sites is coordinated through phase, i.e. when two signals from distant structures are in phase, correlated firing of APs in the two structures is selectively enhanced. For example, a significant portion of neurons in the medial prefrontal cortex (PFC) of freely behaving rats are phase locked - a process in which the phase of two oscillators have a constant relationship - to the hippocampal theta rhythm with APs occurring at specific phases of the hippocampal theta (Fig. 4). In the hippocampal-PFC network the hippocampal APs lead activity in the PFC on average by $50 \mathrm{~ms}$ suggesting directionality from hippocampus to PFC (Siapas et al., 2005). This data indicates that phase locking provides temporal ordering of the excitability windows of phase-locked neurons that enhance the direction of flow of information. In addition, phase-locking by consistently regulating the timing of firing within 
hippocampal and PFC cells could result in spike timing-dependent plasticity and result in the selective strengthening of synapses.

This time-linked firing of neuronal population is dependent upon the degree of coherence between waveforms from various structures. The coupling of theta results in increased firing of APs at the time a rat has to make a decision (decision point) in a spatial task (Jones and Wilson, 2005). The coordination of theta rhythms provides a mechanism through which the relative timing of disparate neural activities can be controlled, allowing specialized brain structures to both encode information independently and to interact selectively according to current behavioral demands. Hippocampal- PFC coherence increases throughout training in parallel with behavioral performance (Sigurdsson et al., 2010). This theta-entrained activity across hippocampal-cortical circuits is likely important for information flow and guiding the plastic changes that underlie the dynamic storage of information across these networks (Siapas et al., 2005; Fries, 2005). Not surprisingly, alteration of coherences has been related to poor cognitive ability in rodents (Lee et al., 2014; O'Reilly et al., 2014).

As with theta oscillation, coherences of gamma oscillations have also been shown to have an important role in learning in rodents. Gamma phase synchrony between the entorhinal cortex and CA1 region predicted correct versus incorrect trials in a delayed non-matching-to-place T-maze task (Yamamoto et al., 2014). In this test rats have to learn to alternate arms in the $t$ T-maze to receive a reward. When the animal reached the decision point in the maze, there was a transient burst of synchronous high gamma $(65-140 \mathrm{~Hz})$ before correct, but not incorrect trials. As a further indication of the role of gamma in memory retrieval, optogenetic inhibition of the circuit targeted to the decision choice point area resulted in a reduction in both gamma synchrony and correct execution of a working-memory-guided behavior task (Yamamoto et al., 2014).

Rats with a history of seizures have deficits in synchronization across the hippocampus and PFC, as measured by spectral coherence (Kleen et al., 2011a). Following repetitive seizures early in life, rats had initial difficulties in learning a delayed-non-match-to-sample memory task but with repeated trials eventually performed as well as controls. During training, these rats showed enhanced CA1-prefrontal theta and gamma coherence compared to non-seizure controls (Kleen et al., 2011b)(Fig. 5). Furthermore, seizure rats showed enhanced CA1-PFC theta coherence in correct trials compared with incorrect trials when long delays were imposed, suggesting increased hippocampal-prefrontal cortex synchrony for the task in this group when memory demand was high. These studies suggest that dynamic compensatory changes in this network and interconnected circuits may underlie cognitive rehabilitation following seizures.

Taken together, there is now a considerable amount of data indicating that following seizures there are significant abnormalities in neuronal network properties with abnormalities in rate and temporal coding of neuronal activity both in local and widespread networks. Since precise temporal organization of neuronal firing activity is believed to be a fundamental aspect of normal information processing, even small errors in the timing of neuronal and oscillatory activities can amplify across complex networks resulting in cognitive dysfunction (Buzsaki, 2007). 


\section{Common mechanisms in seizures and cognitive impairment}

Insights about cognitive comorbidities can be gleamed from studying signaling pathways in the genetic epilepsies. In a number of the genetic epilepsies the mechanisms causing seizure activity are similar to those causing cognitive impairment. In addition, as described above, seizures themselves may further exacerbate cognitive deficits. Two examples of genetic disorders associated with both epilepsy and cognitive dysfunction, tuberous sclerosis complex (TSC) and Dravet Syndrome (DS) will be briefly discussed.

TSC. TSC is an autosomal dominant inherited disease of high penetrance, characterized pathologically by the presence of hamartomas (tumor-like lesions) in multiple organ systems. Well known clinical manifestations include epilepsy, learning difficulties, behavioral problems, and skin lesions. Many patients have renal lesions, usually angiomyolipomas; cysts, polycystic renal disease, and renal carcinoma can also occur. The neurological manifestations of TSC are particularly challenging and include infantile spasms, intractable epilepsy and cognitive disabilities which vary from mild learning disabilities to severe intellectual impairment. While epilepsy in this condition can worsen cognitive function, particularly when the seizures are frequent and occur at a young age, cognitive deficits can occur even in the absence of seizures.

The majority of individuals with tuberous sclerosis complexes have mutations of Tsc1 (encoding for the protein hamartin) or $T_{s c 2}$ (encoding for the protein tuberin). Hamartin and tuberin proteins form a functional complex, which inhibits the serine/threonine protein kinase mammalian target of rapamycin (mTOR). The mTOR kinase complex is the central component of a cell growth pathway that responds to changes in nutrients, energy balance, and extracellular signals to control cellular processes including protein synthesis, energy metabolism, and autophagy (Laplante and Sabatini, 2012). Loss of function of the Tsc1 and $T s c 2$ protein complex results in deregulated and constitutively active mTOR complex 1 , which promotes cell growth and contributes to tumor formation in dividing cells including the hamartomas hamartomas and giant cells that are characteristic of TSC.

Mouse models of TSC exhibit behavioral changes paralleling human disease phenotypes including seizures and deficits in learning and memory (Ehninger et al., 2008; Tsai et al., 2012). However, as in humans, cognitive impairment can occur in the absence of seizures. Heterozygous knockout of either Tsc1 or Tsc2 in mice is sufficient to disrupt hippocampal dependent spatial learning and memory in the Morris water maze task as well as context discrimination in a fear-conditioning assay (Goorden et al., 2007; Ehninger et al., 2008) without causing seizures.

There are a number of mechanisms by which diminished mTOR -dependent translational control could interfere with proper information processing and memory storage and retrieval. While mTOR signaling regulates the synthesis of many proteins positively, it down-regulates other proteins, including Kv1.1. This down-regulation of Kv1.1 would be predicted to increase brain excitability while also impairing memory and learning. Functional mutations in $T s c 1$ or $T s c 2$ significantly alter synapse structure, function and plasticity (Auerbach et al., 2011; Bateup et al., 2011; Chevere-Torres et al., 2012). Protein 
synthesis, regulated by TSC-mTOR signaling, plays a role in learning-associated synaptic changes. In TSC, increased local availability of proteins may stabilize plasticity at synapses that would not normally undergo synaptic consolidation, increasing the signal-to-noise ratio and degrading the specificity of synaptic modifications occurring during normal learning, as evidenced by lower thresholds for the induction of late-phase LTP in several mouse modes of TSC (Ehninger et al., 2008). Rapamycin, a mTor inhibitor, not only reverses abnormal synaptic consolidation but also restores learning deficits in Tsc2+ mice (Ehninger et al., 2008).

While it is clear that perturbations of Tsc1/2 and mTOR alter many aspects of neuronal function, it is unclear which alterations are directly causal and which are induced secondarily as a consequence of altered brain function. To address this issue, Bateup et al. (Bateup et al., 2011) knocked out Tsc1 selectively in CA1 pyramidal neurons in P14-16 mouse pups. The loss of Tsc1 produced hyperexcitable pyramidal cells due to deficits in inhibitory synaptic function manifested as decreased amplitude of miniature inhibitory currents, reduced evoked inhibitory currents and reduced synaptic inhibitory potentials. These studies suggest that Tsc1 is critical for establishing inhibitory synapses onto CA1 pyramidal cells. The overall deficit in inhibitory drive onto pyramidal cells resulted in a disrupted excitatory/inhibition ratio and increased hippocampal network activity both in vivo and in vitro. The observed reduction in inhibition was reversed by blocking mTOR activity with rapamycin, suggesting that amelioration of the signaling abnormality can restore normal cell activity. Thus disrupted development of inhibitory synaptic transmission is likely an important consequence of altering Tsc1/2-mTOR signaling and restoring E/I balance may stabilize normal cell activity. Indeed, artificial elevation of cellular E/I ratio has also been shown to disrupt information processing and social behavior in mice, further suggesting that stabilization of the balance between excitatory and inhibitory drive is important for cognitive function (Yizhar et al., 2011). Maintenance of E/I balance has also been demonstrated to drive normal hippocampal oscillations. In oscillating hippocampal pyramidal cells synaptic excitation is immediately followed by proportional synaptic inhibition, and modification of the amplitude of these events directly modulates oscillation frequency (Atallah and Scanziani, 2009).

Thus the mechanisms responsible for both the epilepsy and cognitive dysfunction appear to occur as a result of an abnormal signaling pattern that results in hyperexcitability and altered synaptic function. All of these pathological features are likely to result in aberrant oscillations, which as described above, can have adverse effects on cognition.

Dravet syndrome (DS) is a life-long epilepsy syndrome with childhood onset that is associated with severe cognitive and quality-of-life impairments (Bender et al., 2012). Intellectual disability in DS begins early in development and is permanent; scores on the developmental quotient typically drop to $20-40 \%$ of normal within the first 6 years of age and, as adults, IQ scores are below 50 in the majority of cases (Bender et al., 2012).

In $85 \%$ of children with DS there is a mutation in the SCN1A gene, resulting in loss of function of the type I voltage-gated sodium channel (Nav1.1). Nav1.1 is one of four sodium channels expressed in the brain that are critical for initiating and propagating APs in 
neurons. The mechanism by which SCN1A loss-of-function mutations cause an increased susceptibility to seizures has been investigated with a Scn1a knock-out mouse model. Mice that are heterozygous for the Scn1a deletion are susceptible to febrile (hyperthermiainduced) seizures and have spontaneous seizures (Yu et al., 2006; Oakley et al., 2009). In dissociated hippocampal interneurons and pyramidal cells, sodium currents in inhibitory, GABAergic interneurons, but not excitatory pyramidal cells, are substantially reduced, indicating that the ability of interneurons to provide inhibitory input to postsynaptic cells is impaired (Yu et al., 2006). These results suggest that increased neuronal excitability in disorders linked to SCN1A loss-of-function mutations may be caused by a principal impairment in the ability of interneurons to provide appropriate inhibition in neuronal networks, thus leading to an imbalance of excitatory and inhibitory drive in the brain.

There is some controversy as to whether the presence of seizures negatively impacts cognitive development or whether the loss-of-function of Nav1.1 impairs function of neural networks involved in information processing. To replicate some features of DS, Bender et al. (Bender et al., 2013) used siRNA to reduce Nav1.1 expression in the medial septum and diagonal band of Broca (MSDB). The medial septum and MSDB are critical structures for the generation of hippocampal theta rhythm. Reduction of Nav1.1 in the MSDB caused spatial memory impairment without causing seizures. Spatial performance was significantly related to hippocampal theta frequency in the control group, but this relationship was abolished after knockdown of Nav1.1. This effect is consistent with the critical role of the MSDB in regulating hippocampal theta oscillations, but differs in that it represents a dysregulation of the rhythm rather than a permanent suppression of theta oscillations as is seen after MSDB inactivation (McNaughton et al., 2006). These results reveal a critical role for Nav1.1 in the brain for the proper regulation of network oscillations and cognitive function.

In summary, there are many factors that can lead to cognitive impairment in epilepsy. Seizure-induced molecular and cellular changes that contribute to cognitive impairment are many and likely to be cumulative. Recent work, described above, has implicated alterations of brain oscillations to be a pivotal juncture in cognitive impairment. However, understanding how seizures alter the coordination or synchronization across neuronal networks remains a challenge, as does developing epilepsy-specific therapies to reverse or improve cognitive impairment.

\section{Acknowledgments}

Grant Information: This work was supported by the NIH Grants NS074450, NS074450 and NS073083 and the Emmory R. Shapses Research Fund and Michael J. Pietroniro Research Fund (GLH).

\section{Reference List}

Atallah BV, Scanziani M. Instantaneous modulation of gamma oscillation frequency by balancing excitation with inhibition. Neuron. 2009; 62:566-577. [PubMed: 19477157]

Auerbach BD, Osterweil EK, Bear MF. Mutations causing syndromic autism define an axis of synaptic pathophysiology. Nature. 2011; 480:63-68. [PubMed: 22113615] 
Bateup HS, Takasaki KT, Saulnier JL, Denefrio CL, Sabatini BL. Loss of Tsc1 in vivo impairs hippocampal mGluR-LTD and increases excitatory synaptic function. J Neurosci. 2011; 31:88628869. [PubMed: 21677170]

Baumbach HD, Chow KL. Visuocortical epileptiform discharges in rabbits: differential effects on neuronal development in the lateral geniculate nucleus and superior colliculus. Brain Res. 1981; 209:61-76. [PubMed: 7214164]

Bell B, Lin JJ, Seidenberg M, Hermann B. The neurobiology of cognitive disorders in temporal lobe epilepsy. Nat Rev Neurol. 2011; 7:154-164. [PubMed: 21304484]

Benchenane K, Tiesinga PH, Battaglia FP. Oscillations in the prefrontal cortex: a gateway to memory and attention. Curr Opin Neurobiol. 2011

Bender AC, Morse RP, Scott RC, Holmes GL, Lenck-Santini PP. SCN1A mutations in Dravet syndrome: Impact of interneuron dysfunction on neural networks and cognitive outcome. Epilepsy Behav. 2012

Bender AC, Natola H, Ndong C, Holmes GL, Scott RC, Lenck-Santini PP. Focal Scn1a knockdown induces cognitive impairment without seizures. Neurobiol Dis. 2013; 54:297-307. [PubMed: 23318929]

Berg AT, Langfitt JT, Testa FM, Levy SR, DiMario F, Westerveld M, Kulas J. Global cognitive function in children with epilepsy: a community-based study. Epilepsia. 2008; 49:608-614. [PubMed: 18070088]

Berg AT, Zelko FA, Levy SR, Testa FM. Age at onset of epilepsy, pharmacoresistance, and cognitive outcomes: a prospective cohort study. Neurology. 2012; 79:1384-1391. [PubMed: 22972641]

Blake RV, Wroe SJ, Breen EK, McCarthy RA. Accelerated forgetting in patients with epilepsy: evidence for an impairment in memory consolidation. Brain. 2000; 123(Pt 3):472-483. [PubMed: 10686171]

Boukhezra O, Riviello P, Fu DD, Lui X, Zhao Q, Akman C, Holmes GL. Effect of the postictal state on visual-spatial memory in immature rats. Epilepsy Res. 2003; 55:165-175. [PubMed: 12972171]

Butler CR, Bhaduri A, Acosta-Cabronero J, Nestor PJ, Kapur N, Graham KS, Hodges JR, Zeman AZ. Transient epileptic amnesia: regional brain atrophy and its relationship to memory deficits. Brain. 2009; 132:357-368. [PubMed: 19073652]

Butler CR, Zeman AZ. Recent insights into the impairment of memory in epilepsy: transient epileptic amnesia, accelerated long-term forgetting and remote memory impairment. Brain. 2008; 131:2243-2263. [PubMed: 18669495]

Buzsaki G. Theta oscillations in the hippocampus. Neuron. 2002; 33:325-340. [PubMed: 11832222]

Buzsaki G. The structure of consciousness. Nature. 2007; 446:267. [PubMed: 17361165]

Buzsaki G, Draguhn A. Neuronal oscillations in cortical networks. Science. 2004; 304:1926-1929. [PubMed: 15218136]

Carr MF, Karlsson MP, Frank LM. Transient slow gamma synchrony underlies hippocampal memory replay. Neuron. 2012; 75:700-713. [PubMed: 22920260]

Chauviere L, Rafrafi N, Thinus-Blanc C, Bartolomei F, Esclapez M, Bernard C. Early deficits in spatial memory and theta rhythm in experimental temporal lobe epilepsy. J Neurosci. 2009; 29:5402-5410. [PubMed: 19403808]

Chevere-Torres I, Kaphzan H, Bhattacharya A, Kang A, Maki JM, Gambello MJ, Arbiser JL, Santini E, Klann E. Metabotropic glutamate receptor-dependent long-term depression is impaired due to elevated ERK signaling in the DeltaRG mouse model of tuberous sclerosis complex. Neurobiol Dis. 2012; 45:1101-1110. [PubMed: 22198573]

Choy M, Dube CM, Ehrengruber M, Baram TZ. Inflammatory processes, febrile seizures, and subsequent epileptogenesis. Epilepsy Curr. 2014; 14:15-22. [PubMed: 24955071]

Committee on the Public Health Dimensions of the Epilepsies, Board on Health Sciences Policy, Institute of Medicine. Epilepsy Across the Spectrum: Promoting Health and Understanding. The National Academies Press; 2012.

Dupret D, O'Neill J, Pleydell-Bouverie B, Csicsvari J. The reorganization and reactivation of hippocampal maps predict spatial memory performance. Nat Neurosci. 2010; 13:995-1002. [PubMed: 20639874] 
Ego-Stengel V, Wilson MA. Disruption of ripple-associated hippocampal activity during rest impairs spatial learning in the rat. Hippocampus. 2010; 20:1-10. [PubMed: 19816984]

Ehninger D, Han S, Shilyansky C, Zhou Y, Li W, Kwiatkowski DJ, Ramesh V, Silva AJ. Reversal of learning deficits in a Tsc2+/- mouse model of tuberous sclerosis. Nat Med. 2008; 14:843-848. [PubMed: 18568033]

Farwell JR, Dodrill CB, Batzel LW. Neuropsychological abilities of children with epilepsy. Epilepsia. 1985; 26:395-400. [PubMed: 4043009]

Fastenau PS, Jianzhao S, Dunn DW, Austin JK. Academic underachievement among children with epilepsy: proportion exceeding psychometric criteria for learning disability and associated risk factors. J Learn Disabil. 2008; 41:195-207. [PubMed: 18434287]

Faure JB, Marques-Carneiro JE, Akimana G, Cosquer B, Ferrandon A, Herbeaux K, Koning E, Barbelivien A, Nehlig A, Cassel JC. Attention and executive functions in a rat model of chronic epilepsy. Epilepsia. 2014; 55:644-653. [PubMed: 24621352]

Foster DJ, Wilson MA. Reverse replay of behavioural sequences in hippocampal place cells during the awake state. Nature. 2006; 440:680-683. [PubMed: 16474382]

Fries P. A mechanism for cognitive dynamics: neuronal communication through neuronal coherence. Trends Cogn Sci. 2005; 9:474-480. [PubMed: 16150631]

Goorden SM, van Woerden GM, van der Weerd L, Cheadle JP, Elgersma Y. Cognitive deficits in Tsc1+/- mice in the absence of cerebral lesions and seizures. Ann Neurol. 2007; 62:648-655. [PubMed: 18067135]

Gray CM, Viana Di PG. Stimulus-dependent neuronal oscillations and local synchronization in striate cortex of the alert cat. J Neurosci. 1997; 17:3239-3253. [PubMed: 9096157]

Hermann B, Meador KJ, Gaillard WD, Cramer JA. Cognition across the lifespan: antiepileptic drugs, epilepsy, or both? Epilepsy Behav. 2010; 17:1-5. [PubMed: 19931492]

Hernan AE, Alexander A, Jenks KR, Barry J, Lenck-Santini PP, Isaeva E, Holmes GL, Scott RC. Focal epileptiform activity in the prefrontal cortex is associated with long-term attention and sociability deficits. Neurobiol Dis. 2014; 63:25-34. [PubMed: 24269731]

Hyman JM, Wyble BP, Goyal V, Rossi CA, Hasselmo ME. Stimulation in hippocampal region CA1 in behaving rats yields long-term potentiation when delivered to the peak of theta and long-term depression when delivered to the trough. J Neurosci. 2003; 23:11725-11731. [PubMed: 14684874]

Isaeva E, Isaev D, Holmes GL. Alteration of synaptic plasticity by neonatal seizures in rat somatosensory cortex. Epilepsy Res. 2013

Isaeva E, Isaev D, Khazipov R, Holmes GL. Selective impairment of GABAergic synaptic transmission in the flurothyl model of neonatal seizures. Eur J Neurosci. 2006; 23:1559-1566. [PubMed: 16553619]

Isaeva E, Isaev D, Savrasova A, Khazipov R, Holmes GL. Recurrent neonatal seizures result in longterm increases in neuronal network excitability in the rat neocortex. Eur J Neurosci. 2010; 31:1446-1455. [PubMed: 20384780]

Jadhav SP, Kemere C, German PW, Frank LM. Awake hippocampal sharp-wave ripples support spatial memory. Science. 2012; 336:1454-1458. [PubMed: 22555434]

Jones MW, Wilson MA. Theta rhythms coordinate hippocampal-prefrontal interactions in a spatial memory task. PLoS Biol. 2005; 3:e402. [PubMed: 16279838]

Karnam HB, Zhao Q, Shatskikh T, Holmes GL. Effect of age on cognitive sequelae following early life seizures in rats. Epilepsy Res. 2009a; 85:221-230. [PubMed: 19395239]

Karnam HB, Zhou JL, Huang LT, Zhao Q, Shatskikh T, Holmes GL. Early life seizures cause longstanding impairment of the hippocampal map. Exp Neurol. 2009b; 217:378-387. [PubMed: 19345685]

Kleen JK, Scott RC, Holmes GL, Lenck-Santini PP. Hippocampal interictal spikes disrupt cognition in rats. Ann Neurol. 2010; 67:250-257. [PubMed: 20225290]

Kleen JK, Scott RC, Holmes GL, Roberts DW, Rundle MM, Testorf M, Lenck-Santini PP, Jobst BC. Hippocampal interictal epileptiform activity disrupts cognition in humans. Neurology. 2013; 81:18-24. [PubMed: 23685931]

Kleen JK, Scott RC, Lenck-Santini PP, Holmes GL. Cognitive and Behavioral Co-Morbidities of Epilepsy. 2012 
Kleen JK, Sesque A, Wu EX, Miller FA, Hernan AE, Holmes GL, Scott RC. Early-life seizures produce lasting alterations in the structure and function of the prefrontal cortex. Epilepsy Behav. 2011a; 22:214-219. [PubMed: 21873119]

Kleen JK, Wu EX, Holmes GL, Scott RC, Lenck-Santini PP. Enhanced oscillatory activity in the hippocampal-prefrontal network is related to short-term memory function after early-life seizures. J Neurosci. 2011b; 31:15397-15406. [PubMed: 22031886]

Koyama R, Tao K, Sasaki T, Ichikawa J, Miyamoto D, Muramatsu R, Matsuki N, Ikegaya Y. GABAergic excitation after febrile seizures induces ectopic granule cells and adult epilepsy. Nat Med. 2012; 18:1271-1278. [PubMed: 22797810]

Krauss GL, Summerfield M, Brandt J, Breiter S, Ruchkin D. Mesial temporal spikes interfere with working memory. Neurology. 1997; 49:975-980. [PubMed: 9339676]

Laplante M, Sabatini DM. mTOR signaling in growth control and disease. Cell. 2012; 149:274-293. [PubMed: 22500797]

Lee H, Dvorak D, Fenton AA. Targeting Neural Synchrony Deficits is Sufficient to Improve Cognition in a Schizophrenia-Related Neurodevelopmental Model. Front Psychiatry. 2014; 5:15. [PubMed: 24592242]

Lega BC, Jacobs J, Kahana M. Human hippocampal theta oscillations and the formation of episodic memories. Hippocampus. 2012; 22:748-761. [PubMed: 21538660]

Lenck-Santini PP, Holmes GL. Altered phase precession and compression of temporal sequences by place cells in epileptic rats. J Neurosci. 2008; 28:5053-5062. [PubMed: 18463258]

Lin H, Holmes GL, Kubie JL, Muller RU. Recurrent seizures induce a reversible impairment in a spatial hidden goal task. Hippocampus. 2009

Liu X, Muller RU, Huang LT, Kubie JL, Rotenberg A, Rivard B, Cilio MR, Holmes GL. Seizureinduced changes in place cell physiology: relationship to spatial memory. J Neurosci. 2003; 23:11505-11515. [PubMed: 14684854]

Lugo JN, Swann JW, Anderson AE. Early-life seizures result in deficits in social behavior and learning. Exp Neurol. 2014; 256:74-80. [PubMed: 24685665]

Martinos MM, Yoong M, Patil S, Chin RF, Neville BG, Scott RC, de HM. Recognition memory is impaired in children after prolonged febrile seizures. Brain. 2012; 135:3153-3164. [PubMed: 22945967]

McNaughton N, Ruan M, Woodnorth MA. Restoring theta-like rhythmicity in rats restores initial learning in the Morris water maze. Hippocampus. 2006; 16:1102-1110. [PubMed: 17068783]

Montgomery SM, Buzsaki G. Gamma oscillations dynamically couple hippocampal CA3 and CA1 regions during memory task performance. Proc Natl Acad Sci U S A. 2007; 104:14495-14500. [PubMed: 17726109]

Muhlert N, Grunewald RA, Hunkin NM, Reuber M, Howell S, Reynders H, Isaac CL. Accelerated long-term forgetting in temporal lobe but not idiopathic generalised epilepsy. Neuropsychologia. 2011; 49:2417-2426. [PubMed: 21549134]

Myers CE, Bermudez-Hernandez K, Scharfman HE. The influence of ectopic migration of granule cells into the hilus on dentate gyrus-CA3 function. PLoS ONE. 2013; 8:e68208. [PubMed: 23840835]

Neill JC, Liu Z, Sarkisian M, Tandon P, Yang Y, Stafstrom CE, Holmes GL. Recurrent seizures in immature rats: effect on auditory and visual discrimination. Brain Res Dev Brain Res. 1996; 95:283-292. [PubMed: 8874904]

O'Keefe J, Dostrovsky J. The hippocampus as a spatial map: preliminary evidence from unit activity in the freely-moving rat. Brain Res. 1971; 34:171-175. [PubMed: 5124915]

O'Keefe J, Recce M. Phase relationships between hippocampal place units and the EEG theta rhythm. Hippocampus. 1993; 3:317-330. [PubMed: 8353611]

O'Reilly KC, Kao HY, Lee H, Fenton AA. Converging on a core cognitive deficit: the impact of various neuro developmental insults on cognitive control. Front Neurosci. 2014; 8:153. [PubMed: 24966811]

Oakley JC, Kalume F, Yu FH, Scheuer T, Catterall WA. Temperature- and age-dependent seizures in a mouse model of severe myoclonic epilepsy in infancy. Proc Natl Acad Sci U S A. 2009;

106:3994-3999. [PubMed: 19234123] 
Ostrach LH, Crabtree JW, Campbell BG, Chow KL. Effects of bicuculline-induced epileptiform activity on development of receptive field properties in striate cortex and lateral geniculate nucleus of the rabbit. Brain Res. 1984; 317:113-123. [PubMed: 6467026]

Richard GR, Titiz A, Tyler A, Holmes GL, Scott RC, Lenck-Santini PP. Speed modulation of hippocampal theta frequency correlates with spatial memory performance. Hippocampus. 2013

Robbe D, Buzsaki G. Alteration of theta timescale dynamics of hippocampal place cells by a cannabinoid is associated with memory impairment. J Neurosci. 2009; 29:12597-12605. [PubMed: 19812334]

Sayin U, Sutula TP, Stafstrom CE. Seizures in the developing brain cause adverse long-term effects on spatial learning and anxiety. Epilepsia. 2004; 45:1539-1548. [PubMed: 15571512]

Siapas AG, Lubenov EV, Wilson MA. Prefrontal phase locking to hippocampal theta oscillations. Neuron. 2005; 46:141-151. [PubMed: 15820700]

Sigurdsson T, Stark KL, Karayiorgou M, Gogos JA, Gordon JA. Impaired hippocampal prefrontal synchrony in a genetic mouse model of schizophrenia. Nature. 2010; 464:763-767. [PubMed: 20360742]

Singer AC, Carr MF, Karlsson MP, Frank LM. Hippocampal SWR activity predicts correct decisions during the initial learning of an alternation task. Neuron. 2013; 77:1163-1173. [PubMed: 23522050]

Skaggs WE, McNaughton BL, Wilson MA, Barnes CA. Theta phase precession in hippocampal neuronal populations and the compression of temporal sequences. Hippocampus. 1996; 6:149172. [PubMed: 8797016]

Thompson PJ, Corcoran R. Everyday memory failures in people with epilepsy. Epilepsia. 1992; 33(Suppl 6):S18-S20. [PubMed: 1486831]

Thompson PJ, Duncan JS. Cognitive decline in severe intractable epilepsy. Epilepsia. 2005; 46:17801787. [PubMed: 16302858]

Titiz AS, Mahoney JM, Testorf ME, Holmes GL, Scott RC. Cognitive impairment in temporal lobe epilepsy: Role of online and offline processing of single cell information. Hippocampus. 2014

Tsai PT, Hull C, Chu Y, Greene-Colozzi E, Sadowski AR, Leech JM, Steinberg J, Crawley JN, Regehr WG, Sahin M. Autistic-like behaviour and cerebellar dysfunction in Purkinje cell Tsc1 mutant mice. Nature. 2012; 488:647-651. [PubMed: 22763451]

Tyler AL, Mahoney JM, Richard GR, Holmes GL, Lenck-Santini PP, Scott RC. Functional network changes in hippocampal CA1 after status epilepticus predict spatial memory deficits in rats. $\mathrm{J}$ Neurosci. 2012; 32:11365-11376. [PubMed: 22895719]

Urrestarazu E, Jirsch JD, Levan P, Hall J, Avoli M, Dubeau F, Gotman J. High-frequency intracerebral EEG activity (100-500 Hz) following interictal spikes. Epilepsia. 2006; 47:1465-1476. [PubMed: 16981862]

Villeneuve N, Ben-Ari Y, Holmes GL, Gaiarsa JL. Neonatal seizures induced persistent changes in intrinsic properties of CA1 rat hippocampal cells. Ann Neurol. 2000; 47:729-738. [PubMed: 10852538]

Wilson MA, McNaughton BL. Reactivation of hippocampal ensemble memories during sleep. Science. 1994; 265:676-679. [PubMed: 8036517]

Womelsdorf T, Schoffelen JM, Oostenveld R, Singer W, Desimone R, Engel AK, Fries P. Modulation of neuronal interactions through neuronal synchronization. Science. 2007; 316:1609-1612. [PubMed: 17569862]

Yamamoto J, Suh J, Takeuchi D, Tonegawa S. Successful execution of working memory linked to synchronized high-frequency gamma oscillations. Cell. 2014; 157:845-857. [PubMed: 24768692]

Yizhar O, Fenno LE, Prigge M, Schneider F, Davidson TJ, O'Shea DJ, Sohal VS, Goshen I, Finkelstein J, Paz JT, Stehfest K, Fudim R, Ramakrishnan C, Huguenard JR, Hegemann P, Deisseroth K. Neocortical excitation/inhibition balance in information processing and social dysfunction. Nature. 2011; 477:171-178. [PubMed: 21796121]

Yu FH, Mantegazza M, Westenbroek RE, Robbins CA, Kalume F, Burton KA, Spain WJ, McKnight GS, Scheuer T, Catterall WA. Reduced sodium current in GABAergic interneurons in a mouse model of severe myoclonic epilepsy in infancy. Nat Neurosci. 2006; 9:1142-1149. [PubMed: 16921370] 
Zhou JL, Lenck-Santini PP, Holmes GL. Postictal Single-cell Firing Patterns in the Hippocampus. Epilepsia. 2007a; 48:713-719. [PubMed: 17437414]

Zhou JL, Lenck-Santini PP, Zhao Q, Holmes GL. Effect of interictal spikes on single-cell firing patterns in the hippocampus. Epilepsia. 2007b; 48:720-731. [PubMed: 17284294]

Zhou JL, Shatskikh TN, Liu X, Holmes GL. Impaired single cell firing and long-term potentiation parallels memory impairment following recurrent seizures. Eur J Neurosci. 2007c; 25:3667-3677. [PubMed: 17610586] 

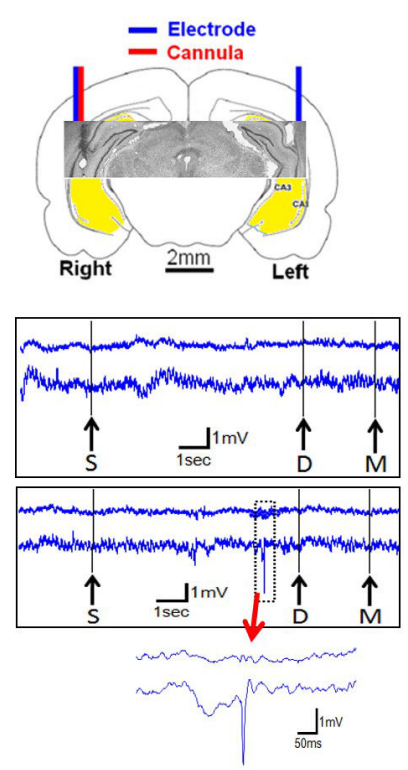

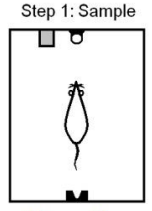

Encoding
Step 2: Delay

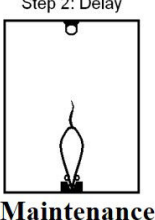

Maintenance

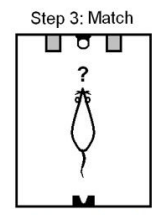

Retrieval

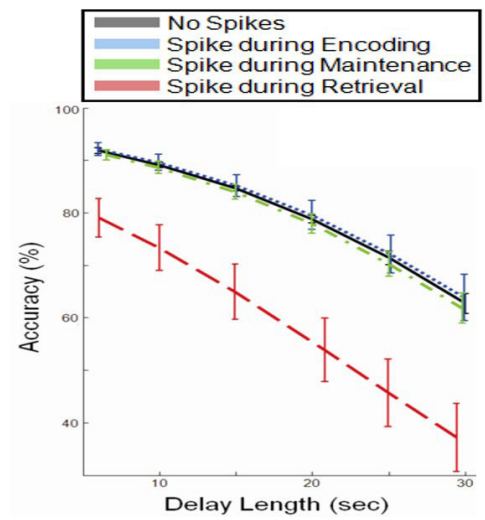

Fig. 1.

To assess the effects of interictal spikes on memory rats underwent intra-hippocampal pilocarpine injections to induced SE. Bilateral electrodes were placed in the ventral hippocampus (A). In the Delayed-Match-To-Sample test during the sample step, one of two levers is randomly presented (right or left) and is pressed by the rat (B). Then, in the Delay step, the rat has to poke its nose into a hole in the opposite wall for a random length of time (6-30 seconds). After this time period has elapsed, the first nosepoke into the hole turns off the stimulus light above and extends both levers. Then, in the Match step, the rat has to remember which lever he pressed during the sample phase, and press that same lever again to procure a food reward. During the sampling stage memory is encoded, during the delay phase memory is maintained, and during the match phase memory is retrieved. Performance is recorded for trials without spikes $(\mathrm{C}$, top trace) and trials with spikes $(\mathrm{C}$, bottom). Among trials in which an IIS occurs during the encoding or maintenance epoch of short-term memory, accuracy does not differ from trials without IIS (D). However, IISs during the retrieval phase produces a marked decrease in accuracy. Increasing delays produce decreases in accuracy, regardless of IIS epoch timing. Modified from Kleen et al., (Kleen et al., 2010) with permission. . 

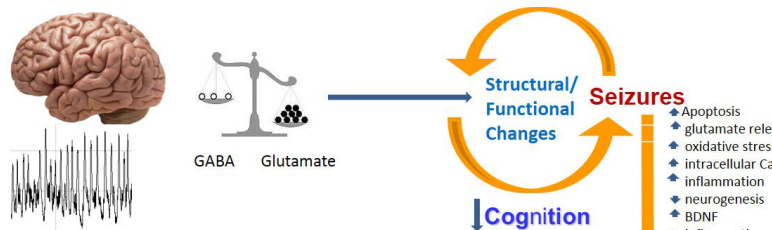

Changes 10 : Apoptosis

GABA Glutamate

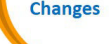

* oxidative stress

* inflammation

$*$ neurogenesis
BDNF

Chronic: days, weeks,

lcognition

- inflammatio

Internalization of GABA receptors

- gaBA currents in hippo

nonths, vears

- in AMPA and NMDA receptors

- oxidative stress
intracellular $\mathrm{Ca}^{2}+$

- Impairment in spike frequency adaptatio

- inflammation

* Impaired long-term potentiation (LTP)

Acute: seconds, min, hours

- necrosis/apoptosis

- GABA transmission

Aneurogenesis
Sprouting

| Cognition

Semi-acute-chronic: hours, days

$\triangle$ in subunit composition of glutamate, AMPA, kainate and GABA receptors

Molecular/ffunctional $\Delta$ in voltage -gated $\mathrm{Na}^{+} \mathrm{Ca}^{2+}$ -

Inflammation

(x)

TGF $\beta$, ErbB-, Wnt- and mTOR signaling, and regulation of actin skeleton

Semi-acute-chronic: days, weeks, months

Fig. 2.

Schematic of SE induced changes that occur over seconds to months. Similar changes can occur with spontaneous seizures. 


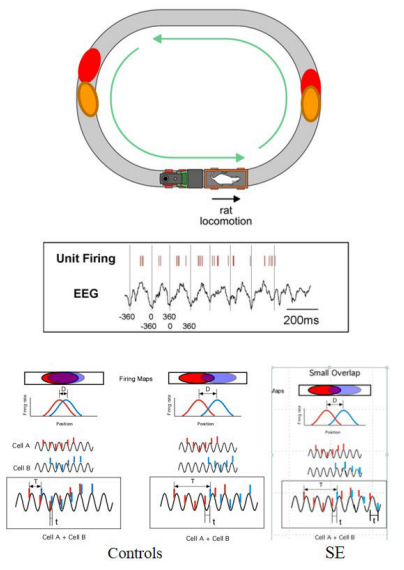

A
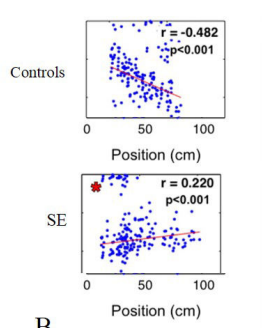

B

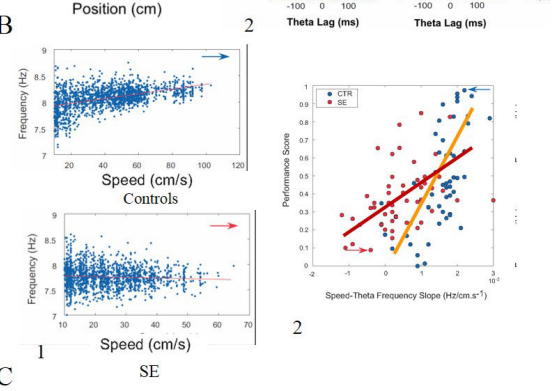

1

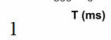

CTR

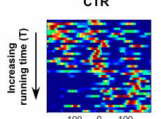

SE

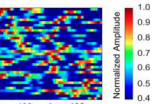

$-1+\frac{1}{10}$

.

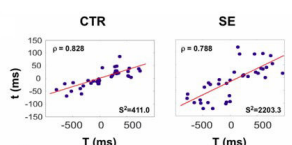

Fig. 3.

Schematic representation of SE induced changes in theta and single cell firing. A. As rat runs around the track several place cells fire. Place cells with partially overlapping place fields (red and blue) will show a different firing relationship on whether the field centers are distant or close to each other. There is a linear relationship between the inter-field distance and the time required to go from the field center to another (running time, T). Because each cell firing is doing phase precession (middle trace), there is also a relationship between the running time (T) and the time interval between APs within the same theta cycle (t). Cells with close fields will fire at short time interval whereas cells with distant fields will fire at long time interval. In the SE rats normal phase precession does not occur and the time interval between APs (t) is variable. As shown in $\mathrm{B}$, the control rat has phase precession whereas the SE rats show phase procession. This abnormality in phase precession is reflected in an abnormal compression of temporal sequences in SE rats. Compared to control pairs of neurons (B1), which show a strong correlation between running-time (T) and thetatime lags ( $t$ ), SE pairs showed a greater variability as expressed by the total variance about the regression line. Cross correlograms for all control and SE pairs ordered in increasing running time (from -1000 ms to $1000 \mathrm{~ms}$ ) (B2). Each horizontal line represents the crosscorrelogram of an individual pair of place cells with the amplitude represented by a color code. In control pairs, the diagonal band near the zero lag line shifts as running-time lag increases. In contrast, this pattern is much less obvious in SE pairs. C. Rats with SE also have impaired speed-theta frequency correlations. Whereas control rats have an increase in frequency of theta with increasing speed, this is not the case with the SE rats (C1). As shown in the right panel $(\mathrm{C} 2)$, performance in a spatial memory task is related to the speedfrequency relationship with SE rats with a poor speed-theta frequency showing impaired performance compared to controls. Modified from Lenck-Santini et al. (Lenck-Santini and Holmes, 2008) and Richard et al. (Richard et al., 2013) with permission. 


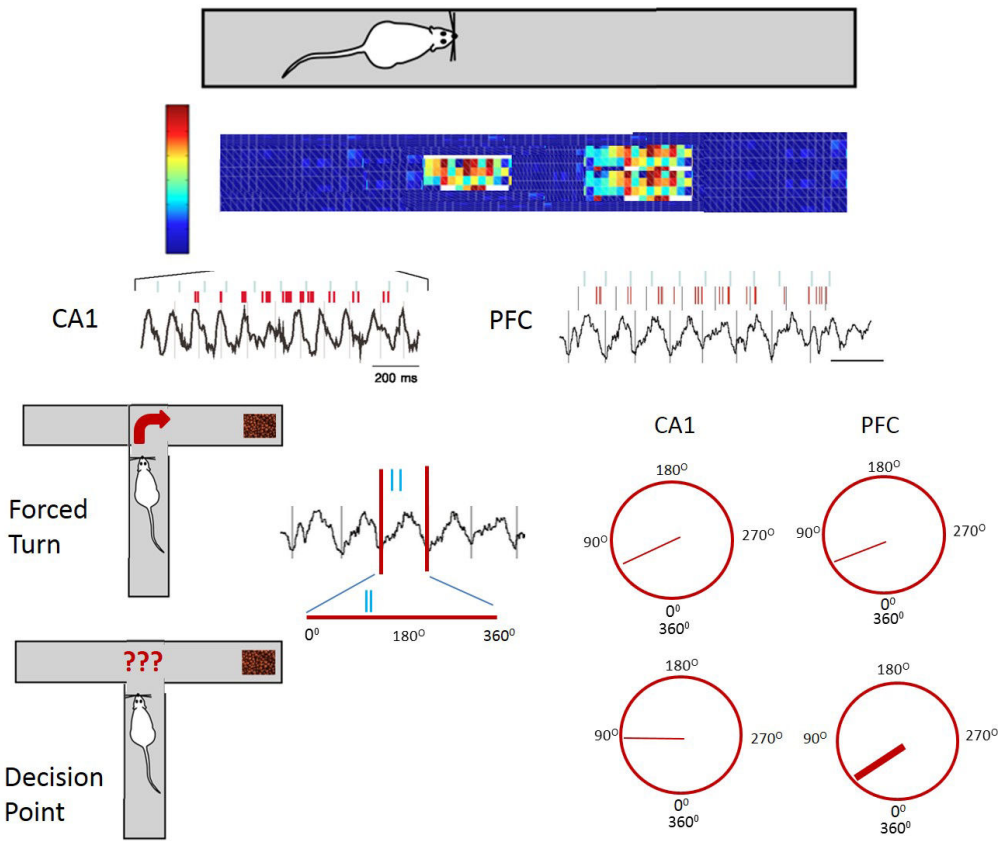

Fig. 4.

Example of hippocampal theta modulation of PFC units. Rat is running on a linear track. Two place cells from the hippocampus are recorded as evidenced by increased firing rates of cells as the animal ran through the place fields. APs from the cells are indicated by vertical blue and red lines positioned above the theta rhythm. In the PFC the APs are temporally linked to the APs in the hippocampus although there is a time delay between the APs in the hippocampus and those in the PFC. The modulation of PFC APs by hippocampal theta occurs even in the absence of PFC theta. In a T-maze animals have to make either a forced turn (top figure) or decided whether to go left or right to receive a food award (cocoa puffs). The theta wave can be drawn as both a straight line as well as a circle to represent the 3600 of the theta wave. When the animal has a forced turn there is a preferred phase (straight line within the circle) for the APs to occur in both CA1 and PFC. When the animal has to make a decision there is a much stronger phase lock of PFC neurons with the hippocampal theta as evidenced by a thicker line. Figures are based on work by Siapas et al. (Siapas et al., 2005) and Jones et al. (Jones and Wilson, 2005). 


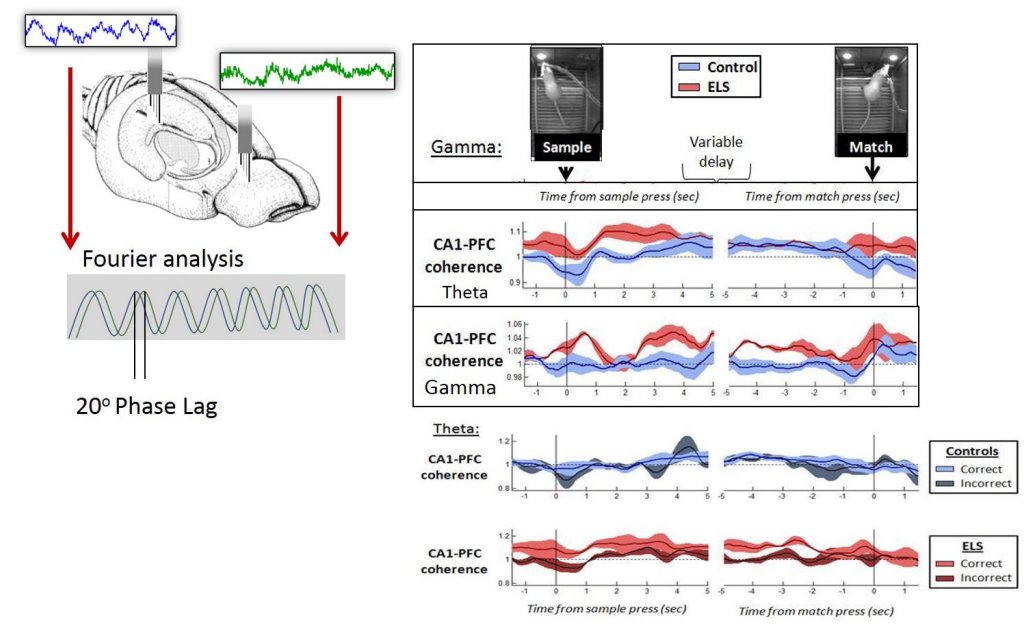

Fig. 5.

Relationship of coherence with cognition. EEG spectral coherence represents the consistency of the phase difference between two EEG signals when compared over time. In a delayed non-match to sample study in rats with a history seizures electrodes were placed in the PFC and hippocampus. Coherences between the waveforms in the hippocampus and PFC were calculated. Coherence is a measure of synchronization or coupling between two EEG signals and is based mainly on the conformity of phase differences between the EEG signals. The two waves shown from the PFC and hippocampus are of the same amplitude and frequency, but there is a phase shift. Phase differences are typically measured in degrees where a complete cycle is 360 degrees. In this example, the phase difference is approximately 30 degrees. Coherences are dynamically measured when controls and rats with early-life seizures (ELS) are doing the test. Data on left is time-linked to the sample press, and data on the right to the match press, revealing functional differences between control (blue) and ELS (red) rats when the envelopes diverge. ELS rats showed increased theta and gamma coherences following the sample press. The lower panel show dynamic CA1-PFC theta coherence in correct and incorrect trials. In trials with 20-30 second delays, control rats did not show performance-related differences in CA1-PFC coherence between correct (light blue) and incorrect (dark blue). In trials with 20-30 second delays, ELS rats showed increased CA1-PFC coherence in correct trials (light red) relative to incorrect trials (dark red) particularly around the time of the sample press and prior to the match press (arrows). Modified from Kleen et al. (Kleen et al., 2011b) with permission. 